Research Article

\title{
Omega Index of Line and Total Graphs
}

\author{
Musa Demirci $\mathbb{D}^{1},{ }^{1}$ Sadik Delen $\mathbb{D}^{2},{ }^{2}$ Ahmet Sinan Cevik $\mathbb{D}^{3},{ }^{3}$ and Ismail Naci Cangul $\mathbb{D}^{1}$ \\ ${ }^{1}$ Faculty of Arts and Science, Department of Mathematics, Bursa Uludag University, Bursa 16059, Turkey \\ ${ }^{2}$ Mathematics Department, Uludag University, Bursa, Turkey \\ ${ }^{3}$ Faculty of Science, Department of Mathematics, Selcuk University, Konya, Turkey
}

Correspondence should be addressed to Ismail Naci Cangul; cangul@uludag.edu.tr

Received 7 February 2021; Accepted 9 September 2021; Published 27 September 2021

Academic Editor: Elena Guardo

Copyright (C) 2021 Musa Demirci et al. This is an open access article distributed under the Creative Commons Attribution License, which permits unrestricted use, distribution, and reproduction in any medium, provided the original work is properly cited.

A derived graph is a graph obtained from a given graph according to some predetermined rules. Two of the most frequently used derived graphs are the line graph and the total graph. Calculating some properties of a derived graph helps to calculate the same properties of the original graph. For this reason, the relations between a graph and its derived graphs are always welcomed. A recently introduced graph index which also acts as a graph invariant called omega is used to obtain such relations for line and total graphs. As an illustrative exercise, omega values and the number of faces of the line and total graphs of some frequently used graph classes are calculated.

\section{Introduction}

Let $G$ be a simple graph with $V(G)=\left\{v_{1}, v_{2}, \ldots, v_{n}\right\}$ and $E(G)=\left\{e_{1}, e_{2}, \ldots, e_{m}\right\}$ as the vertex and edge sets. $n$ and $m$ are called the order and size of $G$, respectively, and are the most important graph parameters. If $e=u v \in E(G)$, we say that $u$ and $v$ are adjacent and $e$ is incident to $u$ and $v$. The number of edges incident to a vertex $v$ is called the degree of $v$ and denoted by $d_{G} v$, or by $d v$ if there is no confusion. A vertex of degree 1 is named as the pendant vertex. The set of degrees,

$$
\left\{1^{\left(a_{1}\right)}, 2^{\left(a_{2}\right)}, \ldots, \Delta^{\left(a_{\Delta}\right)}\right\}
$$

of all vertices where $\Delta$ is the biggest vertex degree in $G$, is called the degree sequence of the graph.

A graph which is connected and has no cycles is called a tree. A graph is called acyclic, unicyclic, bicyclic, tricyclic, etc. according to the number of cycles it has as $0,1,2,3$, etc. As usual, the path, cycle, star, complete, complete bipartite, and tadpole graphs are denoted by $P_{n}, C_{n}, S_{n}, K_{n}, K_{r, s}$, and $T_{r, s}$, respectively. For other graph theoretical notions used in this paper, see, e.g., [1-3].

Given a graph $G$, the line graph $L(G)$ of $G$ is the graph whose vertex set is $E(G)$ with two vertices of $L(G)$ being adjacent iff corresponding edges in $G$ are adjacent. For some applications of the line graph, see, e.g., $[4,5]$. Similarly, the total graph $T(G)$ of $G$ is the graph whose vertex set is $V(G) \cup E(G)$ with two vertices of $T(G)$ being adjacent iff the corresponding elements of $G$ are either adjacent or incident. Line graphs and total graphs are two examples of derived graphs. Minimal doubly resolving sets and strong metric dimension of the layer sun graph and the line graph of this graph are calculated in [4]. The classical meanness property of some graphs based on line graphs was considered in [5]. For some recent applications of the total graphs, see, e.g., [6-8].

According to definitions, the degree sequences of the line and total graphs are

$$
\begin{aligned}
& D S(L(G))=\left\{d_{G} u+d_{G} v-2: u v \in E(G)\right\}=\left\{d_{G} e: e \in E(G)\right\}, \\
& D S(T(G))=\left\{d_{T(G)} v: v \in V(G)\right\} \cup\left\{d_{T(G)} e: e \in E(G)\right\} .
\end{aligned}
$$

\section{Omega Index and Fundamentals}

In this paper, we study the line and total graphs in relation with omega index and the number of faces known as the 
cyclomatic number. Omega index is an additive quantity defined for a given degree sequence (1) or for a graph with

$$
\Omega(G)=\sum_{i=1}^{\Delta} a_{i}\left(d_{i}-2\right) .
$$

It is shown that $\Omega(G)=2(m-n)$ and therefore it is always an even number. It is shown that the omega characteristic gives us very powerful information about cyclicness and connectedness of all the realizations of a given degree sequence (see, e.g., [9]). In brief, it is shown that all realizations of a degree sequence $D$ with $\Omega(D) \leq-4$ must be disconnected; each connected realization of a degree sequence $D$ with $\Omega(D)=-2$ must be a tree; each connected realization of a degree sequence $D$ with $\Omega(D)=0$ must be a unicyclic graph; each connected realization of a degree sequence $D$ with $\Omega(D)=2$ must be a bicyclic graph, etc. Also, the number of faces of a graph or all the realizations of a given degree sequence is formulated as

$$
r(G)=\frac{\Omega(G)}{2}+c(G)
$$

where $c(G)$ is the number of components of $G$. For more properties of the omega index, see $[10,11]$. The effect of edge and vertex deletion on the omega index is studied in [9]. Next, we obtain the number of pendant vertices of a caterpillar tree which consists of a main path so that all vertices are having maximum distance 1 from the path.

Theorem 1. Let $G$ be a caterpillar tree. Let the nonpendant vertices of $G$ be $v_{1}, v_{2}, \ldots, v_{k}$ so that $v_{1}$ has unique nonpendant neighbor $v_{2}$; $v_{k}$ has unique nonpendant neighbor $v_{k-1}$; and $v_{i}$ has two nompendant vertices $v_{i-1}$ and $v_{i+1}$ for $i=2,3, \ldots, k-1$. The following relation holds:

$$
a_{1}(G)=\sum_{i=1}^{k} d_{G} i-2(k-1),
$$

where $d_{G} i$ is the degree of $v_{i}$.

Proof. By counting, $v_{1}$ has only one nonpendant neighbor and hence $d_{G} 1-1$ pendant neighbors, $v_{k}$ has only one nonpendant neighbor and hence $d_{G} k-1$ pendant neighbors, and similarly, for $i=2,3, \ldots, k-1$, the vertex $v_{i}$ has $d_{G} i-2$ pendant neighbors. Therefore,

$$
\begin{aligned}
a_{1}(G) & =d_{G} 1-1+\sum_{i=2}^{k-1}\left(d_{G} i-2\right)+d_{G} k-1 \\
& =\sum_{i=1}^{k} d_{G} i-2-2(k-2),
\end{aligned}
$$

thus giving the result.

Corollary 1. For any tree $T$, we have

$$
a_{1}(T)+k-m(T)=1 .
$$

For a caterpillar tree, the degree sequence of its line graph can be stated more deterministically.

Theorem 2. Let $T$ be a caterpillar tree. Let the degrees of the nompendant vertices of $T$ be $d_{G} 1, d_{g} 2, \ldots, d_{G} k$. The degree sequence of the line graph of $T$ is

$$
D S(L(T))=\left\{\begin{array}{c}
\left(d_{G} 1-1\right)^{\left(d_{G} 1-1\right)},\left(d_{G} 2-1\right)^{\left(d_{G} 2-2\right)}, \ldots,\left(d_{G}(k-1)-1\right)^{\left(d_{G}(k-1)-2\right)}, \\
\left(d_{G} k-1\right)^{\left(d_{G} k-1\right)},\left(d_{G} 1+d_{G} 2-2\right)^{(1)},\left(d_{G} 2+d_{G} 3-2\right)^{(1)}, \ldots, \\
\left(d_{G}(k-1)+d_{G} k-2\right)^{(1)}
\end{array}\right\}
$$

Proof. By Theorem 5 in [11], there exists a complete graph $K_{d_{G}} i$ in $L(T)$ around each nonpendant vertex $v_{i} \in V(G)$ of degree $d_{T} i$ in $T$. Also, $v_{i} \sim v_{j}$ iff $K_{d_{T}} i$ and $K_{d_{T}} j$ have a common vertex. Therefore, $K_{d_{T}} 1$ has $d_{T} 1-1$ vertices of degree $d_{T} 1-1 ; K_{d_{T}} k$ has $d_{T} k-1$ vertices of degree $d_{T} k-1$; and each of other complete graphs $K_{d_{T}}$ ifor $i=2,3, \ldots, k-1$ has $d_{T} i-2$ vertices of degree $d_{T} i-1$. Also, the intersection vertex of $K_{d_{T}} i$ and $K_{d_{T}}(i+1)$ is of degree $d_{T} i-1+d_{T}(i+1)$ -1 for $i=2,3, \ldots, k-1$. Hence, the result follows.

The next result gives the relation between the edge numbers of $G$ and $L(G)$ by means of the first Zagreb index of $G$ :

Lemma 1. For a given graph $G$, we have

$$
m(L(G))=\frac{1}{2} M_{1}(G)-m(G) .
$$

Proof. By definition, we have

$$
\begin{aligned}
m(L(G)) & =\frac{1}{2} \sum_{e \in E(G)} d e \\
& =\frac{1}{2} \sum_{e=u v \in E(G)}\left(d_{u}+d_{v}-2\right) \\
& =\frac{1}{2} \sum_{u \in V(G)} d_{u}^{2}-\sum_{e \in E(G)} 1 \\
& =\frac{1}{2} M_{1}(G)-m(G) .
\end{aligned}
$$

In the following result, we calculate the omega index of the line graph of $G$ when $G$ is $k$-cyclic by means of triangular numbers $T_{n}=n(n+1) / 2$. 
Corollary 2. For a given graph $G$ with $k$-cycles, we have

$$
\Omega(L(G))=2 \sum_{i=3}^{\Delta}\left(a_{i} T_{i-2}-1\right)+k .
$$

The following results are about the omega index of the line graph.

Theorem 3. For a graph $G$, we have

$$
\Omega(L(G))=M_{1}(G)-4 m(G) .
$$

Proof. As $D S(L(G))=\left\{d_{e_{1}}, d_{e_{2}}, \ldots, d_{e_{m}}\right\}$ and $d_{e_{i}}=d_{u_{i}}+d_{v_{i}}$ -2 , we have

$$
\begin{aligned}
\Omega(L(G)) & =\sum_{e_{i} \in E(G)}\left(d e_{i}-2\right) \\
& =\sum_{u_{i} v_{i} \in E(G)}\left(d_{u_{i}}+d_{v_{i}}-4\right) \\
& =\sum_{u \in V(G)} d_{u}^{2}-4 m(G) \\
& =M_{1}(G)-4 m(G) .
\end{aligned}
$$

Corollary 3. For a graph $G$, we have

$$
\Omega(L(G))=\sum_{i=1}^{n} d_{i}\left(d_{i}-2\right) .
$$

Proof. By Theorem 3,

$$
\begin{aligned}
\Omega(L(G)) & =M_{1}(G)-4 m(G) \\
& =\sum_{i=1}^{n} d_{i}^{2}-2 \sum_{i=1}^{n} d_{i},
\end{aligned}
$$

thus giving the result.

Corollary 4. For a graph $G$, we have

$$
\Omega(L(G))=M_{1}(G)-2 \Omega(G)-4 n(G) .
$$

Proof. It follows from $\Omega(G)=2(m(G)-n(G))$.

Now, we obtain some results on the omega index of the total graphs.

Corollary 5. For a connected $k$-cyclic graph $G$, we have

$$
\Omega(T(G))=M_{1}(G)+2 k-2 .
$$

Corollary 6. For a connected $k$-cyclic graph $G$, we have

$$
\Omega(T(G))=\Omega(L(G))+4 m(G)+2 k-2 .
$$

Proof. It follows by Corollary 5 and Theorem 3.
Corollary 7. For any graph $G$, we have

$$
\Omega(T(G))-\Omega(L(G))=3 \Omega(G)+4 n(G) .
$$

Proof. It follows by the definition of omega index.

This means that for every graph $G$, $\Omega(T(G))-\Omega(L(G))-3 \Omega(G)$ is fixed and is equal to $4 n(G)$.

In [11], the number of faces of the line graph of a tree $T$ was given by

$$
r(L(T))=\sum_{i=3}^{\Delta} a_{i} T_{i-2},
$$

where $T_{n}=n(n+1) / 2$ is the $n$ - th triangular number. In [?], this number for a tricyclic graph $G$ was given by

$$
r(L(G))=3+\sum_{i=3}^{\Delta} a_{i} T_{i-2} .
$$

These suggest the following generalization.

Theorem 4. Let $G$ be a simple, connected, and $k$-cyclic graph with degree sequence (1). The number of faces of the line graph $L(G)$ is

$$
r(L(G))=k+\sum_{i=3}^{\Delta} a_{i} T_{i-2}
$$

Proof. For acyclic part of $G$, the formula is given in equation (20). For each $t$ - cycle $C$ in $G, L(G)$ has another $t$-cycle $C^{\prime}$ formed by joining the midpoints of the edges of $C$. Hence, the number of cycles in $G$ must be added to $\sum_{i=3}^{\Delta} a_{i} T_{i-2}$ giving the result.

Inverse problems in mathematics are quite important due to their applications. In graph theory, the inverse problem is the one which deals with finding the values of a given topological graph index. Here, we solve a similar problem for the number of faces of the line graph.

Theorem 5. Let $G$ be a connected graph. Then, $r(L(G))$ can take any positive integer value.

Proof. By Theorem 4, we have equation (22) for a simple, connected, $k$-cyclic graph $G$. This gives us the following linear equality:

$$
r(L(G))=k+a_{3}+3 a_{4}+6 a_{5}+10 a_{6}+\cdots+\frac{(\Delta-2)(\Delta-1)}{2} a_{\Delta} .
$$

As $a_{i} \geq 0$ are integers and as $a_{3}$ has coefficient $1, r(L(G))$ can take any positive integer value.

Some special cases are as follows. 
Corollary 8. Let $T$ be a tree with no vertices of degree 3 . Then, $r(L(T))$ can take all positive integers except $1,2,4,5,7,8,11$, 14 , and 17.

Proof. By the equality in Theorem 11 in [12], we have

$$
r(L(T))=3 a_{4}+6 a_{5}+10 a_{6}+\cdots+\frac{(\Delta-2)(\Delta-1)}{2} a_{\Delta} .
$$

As $a_{i} \geq 0$ are integers, the result follows.

Corollary 9. Let $T$ be a tree with no vertices of degree 3 or 4. Then, $r(L(T))$ can take all positive integer values except $1,2,3,4,5,7,8,9,11,13,14,17,19,23$, and 29.

Proof. Note that

$$
r(L(T))=6 a_{5}+10 a_{6}+15 a_{7}+\cdots+\frac{(\Delta-2)(\Delta-1)}{2} a_{\Delta} .
$$

After easy observations, we find that the class $10+10 n$ has all integers $\geq 10$ ending with $0 ; 6+10 n$ has all integers $\geq 6$ ending with $6 ; 2 \cdot 6+10 n$ has all integers $\geq 12$ ending with 2 ; $3 \cdot 6+10 n$ has all integers $\geq 18$ ending with $8 ; 21+10 n$ has all integers $\geq 21$ ending with $1 ; 21+2 \cdot 6+10 n$ has all integers $\geq 33$ ending with $3 ; 6 \cdot 4+10 n$ has all integers $\geq 24$ ending with $4 ; 15+10 n$ has all integers $\geq 15$ ending with $5 ; 15+2$. $6+10 n$ has all integers $\geq 27$ ending with 7 ; and finally, $21+$ $3 \cdot 6+10 n$ has all integers $\geq 39$ ending with 9 . This implies that only the values given above cannot be attainable by $r(L(G))$.

Then, we obtain the following result.

Corollary 10. We have

$$
\Omega(L(G))-\Omega(G)=2 \sum_{\Delta} a_{i} T_{i-2}
$$

The following variation of this result has useful applications related to cyclicness.

Corollary 11. We have

$$
r(L(G))=\frac{\Omega(G)}{2}+1+\sum_{i=3}^{\Delta} a_{i} T_{i-2} .
$$

By Corollary 11, we have the following cases:

(i) If $G$ is acyclic, then $r(L(G))=\sum_{i=3}^{\Delta} a_{i} T_{i-2}$

(ii) If $G$ is unicyclic, then $r(L(G))=1+\sum_{i=3}^{\Delta} a_{i} T_{i-2}$

(iii) If $G$ is bicyclic, then $r(L(G))=2+\sum_{i=3}^{\Delta} a_{i} T_{i-2}$

(iv) If $G$ is $k$-cyclic, then $r(L(G))=k+\sum_{i=3}^{\Delta} a_{i} T_{i-2}$

\section{Omega and $r$ of the Line Graphs of Some Special Graphs}

Now, we consider the omega and $r$ values of some frequently used graph classes. First, we give a new proof of the fact that the line graph of $P_{n}$ is $P_{n-1}$.

Lemma 2. We have

$$
L\left(P_{n}\right)=P_{n-1}
$$

Proof. Recall that $D S\left(P_{n}\right)=\left\{1^{(2)}, 2^{(n-2)}\right\}$. We then have

$$
\begin{aligned}
\operatorname{DS}\left(L\left(P_{n}\right)\right) & =\left\{0^{(0)}, 1^{(0)}, \ldots, 1^{(0)}, 0^{(0)}, 1^{(1)}, 2^{(1)}, \ldots, 2^{(1)}, 1^{(1)}\right\} \\
& =\left\{1^{(2)}, 2^{(n-3)}\right\} \\
& =D S\left(P_{n-1}\right) .
\end{aligned}
$$

Hence, $\Omega\left(L\left(P_{n}\right)\right)=\Omega\left(P_{n-1}\right)=-2$ as path graphs are acyclic. Also, $r\left(L\left(P_{n}\right)\right)=0$.

Secondly, it is obvious that $L\left(C_{n}\right)=C_{n}$. Therefore, $\Omega\left(L\left(C_{n}\right)\right)=\Omega\left(C_{n}\right)=0$ and $r\left(L\left(C_{n}\right)\right)=1$.

Next, we consider the line graph of the star graph $S_{n}$. By the definition of the line graph, $L\left(S_{n}\right)=K_{n-1}$. Hence,

$$
\begin{array}{r}
\Omega\left(L\left(S_{n}\right)\right)=\Omega\left(K_{n-1}\right) \\
=(n-1)(n-4), \\
r\left(L\left(S_{n}\right)\right)=r\left(K_{n-1}\right) \\
=\frac{(n-3)(n-2)}{2} .
\end{array}
$$

For a complete graph $K_{n}$, we have $D S\left(L\left(K_{n}\right)\right)=\left\{(2(n-2))^{(n(n-1) / 2)}\right\} \quad$ implying that $\Omega\left(L\left(K_{n}\right)\right)=n(n-1) / 2 \cdot(2 n-6)=n(n-1)(n-3) \quad$ and $r\left(L\left(K_{n}\right)\right)=(n-2)\left(n^{2}-2 n-1\right)$. In Figure 1, we illustrated the case of $K_{4}$.

For a complete bipartite graph $K_{r, s}, L\left(K_{r, s}\right)$ is a regular graph of degree $r+s-2$. Its degree sequence is $\left\{(r+s-2)^{(r s)}\right\}$. Hence, $\Omega\left(L\left(K_{r, s}\right)\right)=r s(r+s-4)$ and $r\left(L\left(K_{r, s}\right)\right)=r s(r+s-4) / 2+1$.

Finally, consider the tadpole graph $T_{r, s} . L\left(T_{r, s}\right)$ has degree sequence $\quad\left\{1^{(1)}, 2^{(r+s-4)}, 3^{(3)}\right\}$. Hence, $\Omega\left(L\left(T_{r, s}\right)\right)=3-1=2$, and therefore, $r\left(L\left(T_{r, s}\right)\right)=2$.

\section{Omega and $r$ of the Total Graphs of Some Special Graphs}

In this section, we calculate the omega and $r$ values of some frequently used graphs. We first give an important property.

Lemma 3. Let $G$ be a connected graph. Then, $T(G)$ cannot have any pendant vertex.

Proof. Let $v \in V(G)$. Let $e$ be an edge incident to $v$. As $G$ is connected, there is at least one adjacent vertex say $u$, to $v$. In 


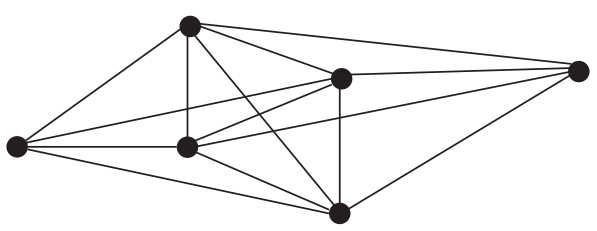

Figure 1: Line graph of $K_{4}$.

the total graph $T(G)$, the vertex $v$ will be adjacent to $u$ and $e$ implying the result.

The proof alternatively follows from the fact that $D S(T(G))$ consists of integers in the form of either $2 d_{i}$ or $e_{j}+2$, where $d_{i}, e_{j} \geq 1$.

Next, we give the relation between omega of $G$ and omega of $T(G)$.

Theorem 6. For a connected graph $G$, we have

$$
\Omega(T(G))=\Omega(G)+M_{1}(G) .
$$

Proof. Recall that $D S(T(G))$ consists of $a_{i}$ times $2 d_{i}$ for every $v_{i} \in V(G)$ and $d e_{j}+2$ 's for every $e_{j}=u_{j} v_{j} \in E(G)$. As $\mathrm{d} e_{j}=\mathrm{d} u_{j}+\mathrm{d} v_{j}-2$, we can deduce that $D S(T(G))$ consists of $a_{i}$ times $2 d_{i}$ for every $v_{i} \in V(G)$ and $\mathrm{d} u_{j}+\mathrm{d} v_{j}$ 's for every $e_{j}=u_{j} v_{j} \in E(G)$. Hence,

$$
\begin{aligned}
\Omega(T(G)) & =\sum_{v_{i} \in V(G)} a_{i}\left(2 d_{i}-2\right)+\sum_{e_{j}=u_{j} v_{j} \in E(G)}\left(\mathrm{d} u_{j}+\mathrm{d} v_{j}-2\right) \\
& =2 m(G)+\Omega(G)+\sum_{v \in V(G)} d_{v}^{2}-2 m(G) \\
& =\Omega(G)+M_{1}(G) .
\end{aligned}
$$

Corollary 11. For a connected graph $G$, we have

$$
\Omega(T(G))=\Omega(G)+\Omega(L(G))+4 m(G) .
$$

Proof. As in Theorem 6, we can write

$$
\begin{aligned}
\Omega(T(G)) & =\sum_{v_{i} \in V(G)} a_{i}\left(2 d_{i}-2\right)+\sum_{e_{j} \in E(G)} \mathrm{d} e_{j} \\
& =2 m(G)+\Omega(G)+\Omega(L(G))+2 m(G) \\
& =\Omega(G)+\Omega(L(G))+4 m(G) .
\end{aligned}
$$

Corollary 12. If $G$ is an acyclic graph, then

$$
\Omega(T(G))=M_{1}(G)-2,
$$

and if $G$ is a unicyclic graph, then

$$
\Omega(T(G))=M_{1}(G) .
$$

Finally, we give the following result for the omega indices of the total graphs of some frequently used graph classes:
Theorem 7. The omega index of the total graphs of some well-known graph classes is as follows:

$$
\begin{aligned}
\Omega\left(T\left(P_{n}\right)\right) & =4 n-8, \\
\Omega\left(T\left(C_{n}\right)\right) & =4 n, \\
\Omega\left(T\left(S_{n}\right)\right) & =(n+1)(n-2), \\
\Omega\left(T\left(K_{n}\right)\right) & =n(n+1)(n-2), \\
\Omega\left(T\left(K_{r, s}\right)\right) & =(r+s)(r s-2)+2 r s, \\
\Omega\left(T\left(T_{r, s}\right)\right) & =4(r+s)+2 .
\end{aligned}
$$

\section{Conclusion}

Derived graphs are graphs obtained from a given graph according to some rules. In this paper, two of the most frequently used derived graphs, the line and total graphs, are studied. Calculating some properties of a derived graph helps to calculate the same properties of the original graph. Here, by means of omega index and several results in recent papers, new relations for line and total graphs are obtained. Also, omega values and the number of faces of the line and total graphs of some frequently used graph classes are calculated. In the future works, similar ideas can be applied to establish several relations for other derived graphs.

\section{Data Availability}

No data were used to support this study.

\section{Conflicts of Interest}

The authors declare that they have no conflicts of interest.

\section{Authors' Contributions}

All authors contributed equally to this paper.

\section{References}

[1] J. A. Bondy and U. S. R. Murty, Graph Theory with Applications, MacMillan, London, UK, 1976.

[2] J. Clark and D. A. Holton, A First Look at Graph Theory, World Scientific, Singapore, 1969.

[3] F. Harary, Graph Theory, Addison-Wesley, Boston, MA, USA, 1969.

[4] J. B. Liu and A. Zafari, "Computing minimal doubly resolving sets and the strong metric dimension of the layer sun graph and the line graph of the layer sun graph," Complexity, vol. 2020, Article ID 6267072, 8 pages, 2020.

[5] G. Muhiuddin, A. M. Alanazi, A. R. Kannan, and V. Govindan, "Preservation of the classical meanness property of some graphs based on line graph operation," Journal of Mathematics, vol. 2021, Article ID 4068265, 10 pages, 2021.

[6] U. Bednarz, I. Wloch, and M. Wolowiec-Musial, "Total graph interpretation of the numbers of the fibonacci type," Journal of Applied Mathematics, vol. 2015, Article ID 837917, 7 pages, 2015.

[7] N. Inayah, I. W. Sudarsana, S. Musdalifah, and N. D. Mangesa, "On super mean labeling for total graph of path and cycle," 
International Journal of Mathematics and Mathematical Sciences, vol. 2018, no. 7-8, 5 pages, Article ID 9250424, 2018.

[8] S. K. Vaidya and D. D. Bantva, "Radio number for total graph of paths," ISRN Combinatorics, vol. 2013, no. 12, 5 pages, Article ID 326038, 2013.

[9] S. Delen, M. Togan, A. Yurttas, U. Ana, and I. N. Cangul, "The effect of edge and vertex deletion on omega invariant," Applicable Analysis and Discrete Mathematics, vol. 14, no. 3, pp. 685-696, 2020.

[10] S. Delen and I. N. Cangul, "Extremal problems on components and loops in graphs," Acta Mathematica Sinica, English Series, vol. 35, no. 2, pp. 161-171, 2019.

[11] H. Ozden, F. Ersoy Zihni, F. Ozen Erdogan, I. N. Cangul, G. Srivastava, and H. M. Srivastava, "Independence number of graphs and line graphs of trees by means of omega invariant," Revista de la Real Academia de Ciencias Exactas, Fisicas y Naturales. Serie A, Matemáticas, vol. 114, no. 2, p. 91, 2020. 